\title{
Análisis del nuevo Módulo de Salud de la Encuesta CASEN 2000
}

\author{
Liliana Jadue $\mathrm{H}^{1}$, Iris D elgado $\mathrm{B}^{2 a}$, Hernán Sandoval $\mathbf{0}^{3}$, \\ Lidia Cabezas $M^{1 b}$, Jeanette Vega $M^{1}$. \\ Analysis of the new health module of \\ the National Socioeconomic \\ Characterization Survey 2000
}

Background: The National Socioeconomic Characterization Survey (CASEN) was modified in the year 2000, to include an assessment of the inequalities in the access to health services. Aim: To analyze the health issues of year 2000 survey database. Material and methods: During 2000, 38338 urban and 26698 rural dwellings were surveyed, totaling 240000 people analyzing ascription to public or private health services and the need demand and use of these services. Results: A higher risk population (lower income, higher age and women) is ascribed to public health services. Sixty five percent of the population self perceives their health as good, this figure decreases along with age and women have a worse self perception than men. In the 30 days prior to the survey, $13 \%$ of the population had a health related event; this figure was higher among women and the lower income quintiles. Expressed health demand was higher among women and lower income quintiles. A multivariate analysis identified an age below 14 years, pertaining to a minority ethnic group, ascription to private health services, residing in rural areas, pertaining to the lower income quintile and male sex, as factors associated to a lack access to health care. In the adjusted model, pertaining to the National Health Fund (a public system) is a protective factor to receive health services. Conclusions: The significant inequalities in the access to health care should be corrected with the new Health Reform. The new module incorporated to the National Socioeconomic Characterization Survey, is usefel to assess the access to health care in Chile (Rev Méd Chile 2004; 132: 750-60). (Key Words: Delivery of healthcare; Health care systems; Health surveys; Socioeconomic factors) Pública, Facultad de Ciencias de la Salud, Universidad del Desarrollo-Clínica Alemana. ${ }^{2}$ Ministerio de Planificación, División de Estudios Sociales. ${ }^{3}$ Comité Interministerial para la Reforma de Salud.

aLicenciada en Matemáticas, Magíster en Bioestadística

bProgramadora en Computación

Correspondencia a: Dra. Liliana Jadue H. Avda. Las Condes

13.800, Santiago. Fono: 56-2-2159330. Fax: 56-5-2159446.

E-mail: ljadue@udd.cl 
$\mathrm{E}$ actual sistema de servicios de salud en Chile es el resultado de la reforma de la seguridad social, que ocumó en 1981, desde un sistema nacional de salud con cobertura universal pública, a un modelo mixto, público y privado, combinando un esquema de seguridad social solidario (público), con un sistema de seguros privados, de carácter competitivo, con base en empresas aseguradoras llamadas ISAPRE (Institución de Salud Previsional) ${ }^{1}$. Hoy día, nuestro sistema de salud enfrenta problemas crecientes, producto de la integración vertical del sector público con el privado que funcionan en forma diferente: el sector público, con una lógica de seguridad social, y el privado con una lógica de seguro individual. Agravan esta situación la segmentación de la población atendida, especialmente en el sector privado de salud, donde el costo anual del seguro es determinado por la ISAPRE sobre la base de tablas de riesgo diferenciales por edad y género, pudiendo ser reajustado de manera unilateral, lo que les ha permitido contar con una cartera de asegurados más jóvenes que el sistema público, que en la práctica actúa como único asegurador de las personas que no acceden a una ISAPRE. De esta forma, las mujeres y los adultos mayores tienen tarifas más altas, debido al mayor riesgo que representan para el asegurador privado de incumir en mayores gastos médicos.

Por esta razón, entre otras, el actual gobierno ha comenzado un nuevo proceso de reforma de salud $^{2}$, que plantea como eje mejorar la equidad de acceso y de nivel de salud.

Para mejorar la situación de equidad en salud de la población chilena, es necesario previamente contar con un sistema de monitoreo y evaluación que diagnostique las inequidades de acceso a atención de salud y su evolución, a medida que se implementa la reforma de salud. Por esto, la Comisión de Reforma, en conjunto con la Iniciativa Chilena para la Equidad en Salud propuso cambios al módulo de salud de la encuesta CASEN en el año 2000, los cuales fueron acogidos por el Ministerio de Planificación y Cooperación (MIDEPLAN). El presente trabajo describe y analiza la situación de equidad de acceso a salud en la población chilena, con base en el módulo de salud reformulado en la encuesta CASEN 2000.

Los objetivos específicos del estudio son:

1. Caracterizar la adscripción a los sistemas de salud previsional de la población chilena en el año 2000.
2. Describir y analizar la necesidad, demanda y utilización de servicios de salud de la población chilena.

\section{MATERIAL Y MÉTODOS}

El estudio se realizó analizando la base de datos de la encuesta CASEN 2000³, solicitada para este efecto a MDEPLAN. Esta encuesta es uno de los instrumentos más importantes que utiliza el gobiemo para evaluar el impacto de las políticas sociales en ejecución en el país, y recoge información sobre educación, salud, vivienda e ingresos de las familias. El marco muestral de la encuesta CASEN se basa en el Censo de Población y Vivienda de 1992. Para definir la muestra, se aplica un muestreo estratificado, por conglomerados y probabilístico sobre el universo de población que habita en hogares particulares del país, excluyendo las zonas de difícil acceso geográfico. Los resultados tienen representación nacional, regional y por zonas urbano/rural. También entrega representatividad comunal en 286 de las 341 comunas del país. El año 2000, la muestra consideró 61.973 viviendas, 37.280 en la zona urbana y 24.693 en la zona rural. Se encuestaron 65.036 hogares, 38.338 en la zona urbana y 26.698 en la zona rural; esto es, aproximadamente, 240.000 personas. El instrumento se aplicó en todo el país, entre noviembre y diciembre del año 2000.

Para caracterizar la adscripción a los sistemas de salud previsional de la población chilena en el año 2000, se utilizó la información de adscripción de acuerdo a sistema público (FONASA), privado (ISAPRE) y otros. La necesidad de atención se evaluó con base en la variable "autopercepción de salud", y las preguntas sobre "necesidad de atención en salud", "demanda expresada de atención de salud" y "demanda no satisfecha de atención de salud". Todas estas variables fueron analizadas por "sexo", "edad", "sistema previsional" y "posición socioeconómica" (quintil de ingresos).

Posteriormente, se ajustó un modelo de regresión logística para estudiar el peso específico de las distintas variables predictoras, en la probabilidad de no satisfacer la demanda de atención de salud, como medida de inequidad de acceso a los servicios. Para el análisis estadístico se utilizó el software SPSS versión 11.0 para Windows. 


\section{RESULTADOS}

Durante el año 2000, 66,5\% de la población se declaró afiliado al sistema público de salud (casi 10 millones de personas) y 19,8\% al sistema de las Instituciones de Salud Previsional ISAPRE (aproximadamente 3 millones de personas). El restante 9,7\% de la población declaró no tener sistema de previsión, sin ser indigentes, 3,2\% pertenecían al sistema de las Fuerzas Armadas y $0,9 \%$ no sabía o no declaró.

La adscripción por sexo mostró diferencias estadísticamente significativas y la presencia de mujeres en FONASA alcanza a $69,4 \%$ versus $64,1 \%$ de los hombres $(p<0,001)$. En las ISAPRE, el porcentaje de hombres fue levemente superior (20,7\% y $19,3 \%$, respectivamente), mientras que en el rubro "Otros" (que incluía a la población en los sistemas de salud de las Fuerzas Armadas y aquellos que no declararon ningún sistema de salud pero que no eran indigentes), la diferencia a favor del sexo masculino estuvo dada principalmente por las Fuerzas Armadas (15,2\% y 11,3\% en hombres y mujeres, respectivamente).

La distribución del sistema previsional de salud por tramos de edad (Figura 1) muestra diferencias al incrementar la edad, especialmente al momento de pasar de la edad laboralmente activa a la edad de jubilación, así, de casi 19\% de adscripción de la población de 45 a 64 años en el sistema privado se redujo a $6,6 \%$ por sobre los 65 años.

El descreme del sector privado también se observó en el análisis de la situación por quintiles de ingreso (Tabla 1). La proporción de adscritos al FONASA superó $80 \%$ en los quintiles más pobres de la población, disminuyendo a 30,6\% en el quintil de mayores ingresos. Análogamente, en la población de ISAPRE, sólo $3,2 \%$ pertenecía al quintil más pobre, y $53,7 \%$ al quinto quintil. Al analizar estos datos en conjunto con la variable sexo, se observó que la proporción de mujeres adscritas al FONASA era mayor en todos los quintiles de ingreso.

Con respecto de la autopercepción en salud, la gran mayoría de los chilenos estimó que su salud es buena o muy buena (65,4\%), y sólo $7 \%$ dijo tener una salud mala o muy mala (Figura 2). En esta tendencia se observan diferencias estadísticamente significativas entre hombre y mujer, nivel de ingreso y tramo de edad. Llama la atención las diferencias por sexo, en que la respuesta positiva de los hombres supera la de las mujeres en casi 10

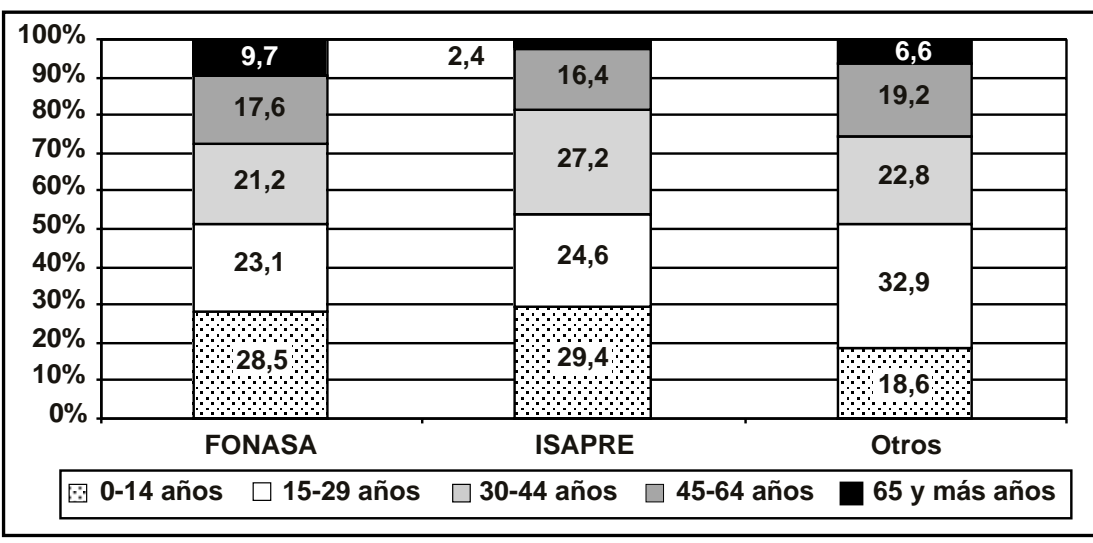

FIguRA 1. Distribución de la población según sistema de previsión y tramos de edad para todo el país. Encuesta CASEN 2000.

Tabla 1. Sistema de Previsión de Salud por quintil de Ingresos. Encuesta CASE N 2000

\begin{tabular}{|lcccc|}
\hline Quintil de Ingresos & FONASA \% & ISAPRE $\%$ & Otro $\%$ & Total $\%$ \\
\hline Quintil I & 87,7 & 3,2 & 9,1 & 100 \\
Quintil II & 80,2 & 8,3 & 11,6 & 100 \\
Quintil III & 67,0 & 18,2 & 14,8 & 100 \\
Quintil IV & 53,1 & 30,2 & 16,7 & 100 \\
Quintil V & 30,6 & 53,7 & 15,7 & 100 \\
Todos & 66,6 & 20,2 & 13,2 & 100 \\
\hline
\end{tabular}


puntos porcentuales, no obstante que la expectativa de vida de las mujeres es 6 años mayor que la de los hombres.

La edad se asocia a una percepción menos positiva del estado de salud. Mientras más de $80 \%$ de los jóvenes consideraron que tienen buena y muy buena salud, sólo 36,9\% de los adultos de 65 y más años declararon lo mismo. En oposición, menos de $2 \%$ de los jóvenes de 15 a 29 años declaró tener una salud mala o muy mala. El análisis por nivel de ingresos y sexo mostró un patrón de comportamiento similar a lo recién descrito, observándose que las mujeres de todos los niveles de ingresos declararon un estado de salud menos satisfactorio que el de los hombres.

La necesidad de servicios de salud se construye a partir de la pregunta: "En los últimos 30 días: tha tenido algún problema de salud, enfermedad o accidente?'. Globalmente, $12,9 \%$ de la población identificó algún evento de salud en ese período. Por sexo, esta necesidad fue mayor en mujeres que en hombres (14,8\% versus 10,9\%). Hay una progre- sión por edad de manera que la población de más de 65 años tuvo casi 4 veces más necesidad de atención de salud que la de 15 años (Tabla 2). Por quintil de ingresos, la necesidad de salud fue similar para todos los grupos, alrededor de $12 \%$.

La Figura 3 muestra la secuencia de análisis que se utilizó, para describir y caracterizar el uso de servicios de salud en la población que tiene necesidad de atención de salud.

El grupo de personas que presentó necesidad de salud (12,9\% de todos los encuestados), fue dividido en dos componentes. El primero se denominó "demanda expresada" y correspondió al $73,4 \%$ de los que tuvieron necesidad. Estos individuos eran los que solicitaban hora para ser atendidos. En este subgrupo se distinguieron dos segmentos: "demanda satisfecha", aquellos que buscaron atención en el sistema de salud tradicional y la obtuvieron; y "demanda no satisfecha", los que no obtuvieron atención por falta de dinero ("barrera financiera"), por falta de acceso geográfico, porque queda muy lejos, está discapacitado, no

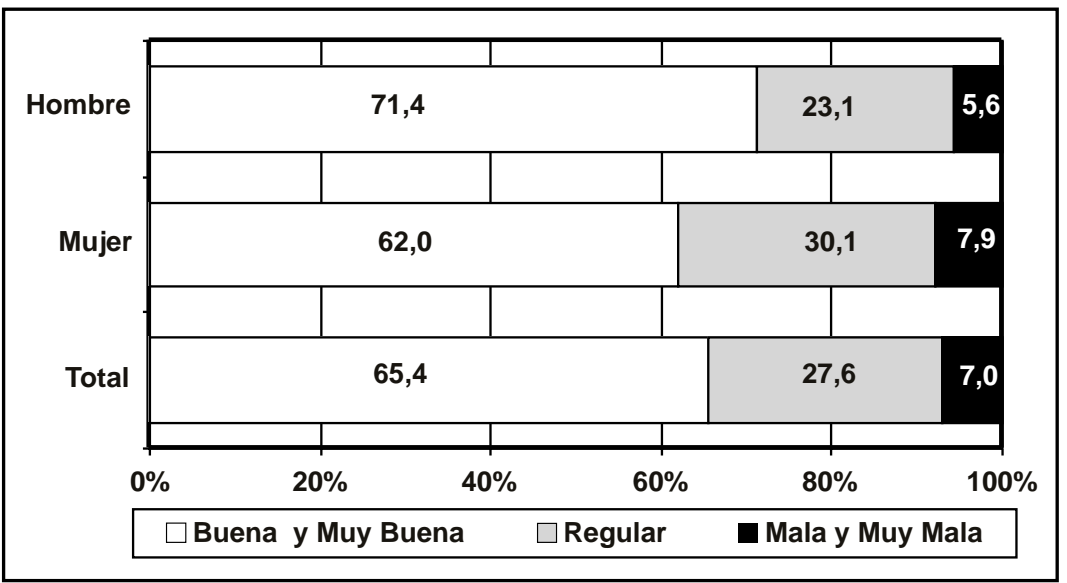

FIgURA 2. Autopercepción de salud por sexo. Encuesta CASEN 2000.

Tabla 2. Necesidad de atención de salud en los 30 días previos por sexo y edad. Encuesta CASEN 2000

\begin{tabular}{|lrrrrrr|}
\hline & \multicolumn{2}{c}{ Hombres } & \multicolumn{2}{c|}{ Mujeres } & \multicolumn{2}{c|}{ Todos } \\
Edad & Si \% & No \% & Si \% & No \% & Si \% & No \% \\
\hline 0-14 años & 13,2 & 86,8 & 12,2 & 87,8 & 12,7 & 87,3 \\
15-29 años & 7,0 & 93,0 & 10,7 & 89,3 & 8,8 & 91,2 \\
30-44 años & 8,5 & 91,5 & 13,6 & 86,4 & 11,2 & 88,8 \\
45-64 años & 12,4 & 87,6 & 20,2 & 79,8 & 16,4 & 83,6 \\
65 y más años & 19,8 & 80,2 & 26,7 & 73,3 & 23,7 & 76,3 \\
Total & 10,9 & 89,1 & 14,8 & 85,2 & 12,9 & 87,1 \\
\hline
\end{tabular}


hay locomoción ("barrera geográfica") o porque pidió hora de atención y no se la dieron ("rechazo del sistema"). El segundo subgrupo entre aquellos que tuvieron un evento de salud (necesidad) es aquél de "demanda no expresada" (26,6\% del universo que tuvo necesidad) que corresponde a las personas que no consideraron necesario consultar, se automedicaron o no consultaron por falta de tiempo, todas razones que hacen suponer que su problema de salud no era grave. A continuación se describen los resultados para cada grupo.

Demanda expresada de atención de salud. Las características por edad y sexo de la demanda expresada muestran que hubo mayor demanda en las mujeres en todas las edades. En ambos sexos, la mayor demanda se presentó en los menores de 14 años con una curva en jota que vuelve a partir de los 45 años de edad.

Según la adscripción previsional, 73,7\% de la población global de FONASA expresó la demanda y $77,9 \%$ en el grupo ISAPRE. La distribución por quintil de ingresos mostró una progresión lineal desde 68,3\% a 79,7\% hacia los quintiles de mayores ingresos, siempre con mayor necesidad en las mujeres.

Demanda satisfecha. Con relación a las formas de satisfacer la demanda, el instrumento consultó por formas tradicionales (en servicios médicos de salud públicos o privados) y no tradicionales de atención, como la consulta en la farmacia, especialistas en medicina alternativa, homeópata $u$ otros lugares. Aproximadamente 3\% de esta población recurrió a los métodos no tradicionales, y por ello el análisis de la demanda satisfecha consideró sólo a las personas atendidas en servicios de salud tradicionales.

La distribución por edad y sexo de la demanda satisfecha (91,8\% de los que expresaron la necesidad de servicios de salud, Figura 3), se observa en la Figura 4, con mayor demanda en las personas de 0 a 14 años en ambos sexos, en una curva en jota que vuelve a elevarse a partir de los 45 años de edad. Sólo en el grupo de 15 a 29 años se observaron diferencias por sexo, con mayor satisfacción de la demanda en las mujeres.

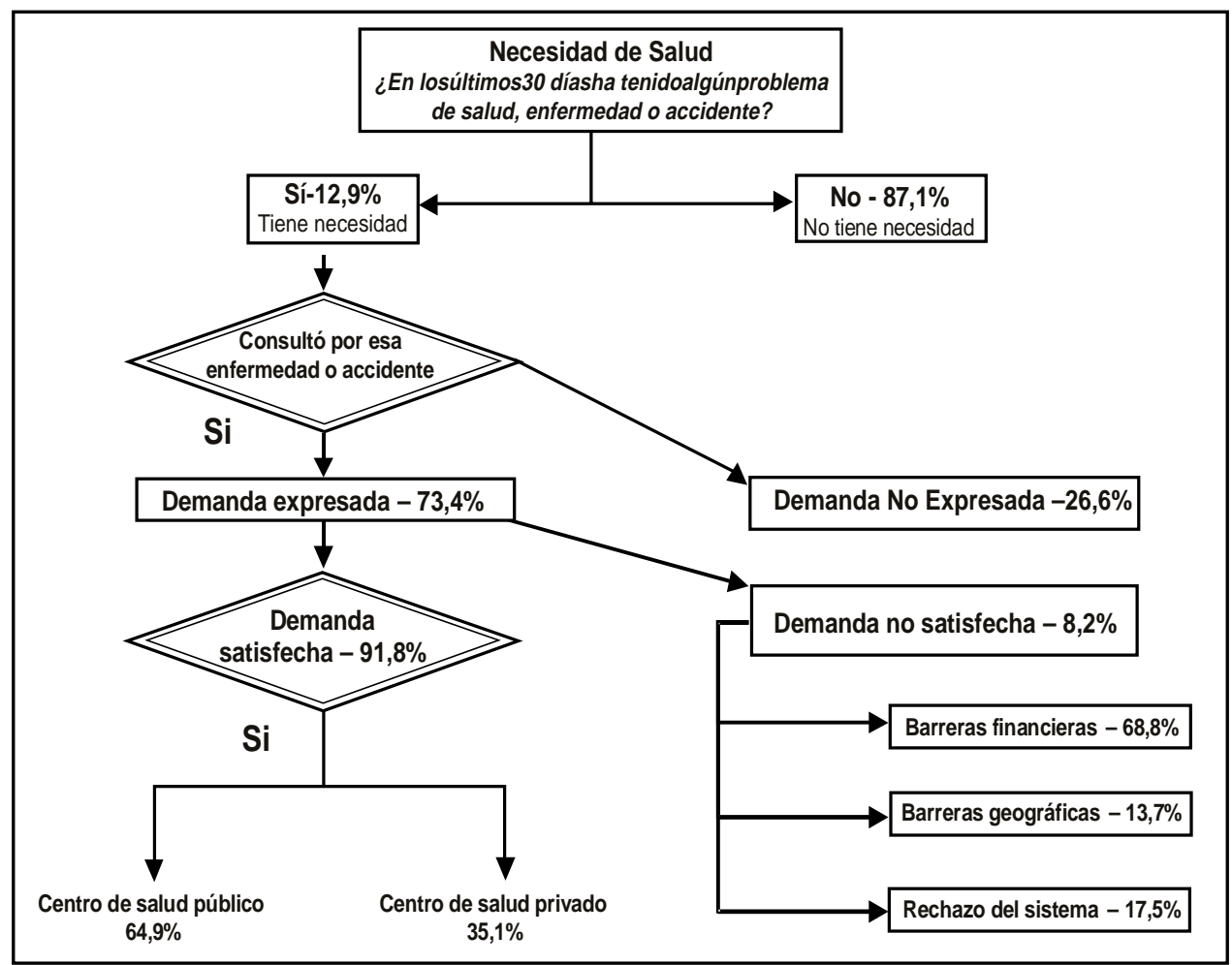

FIguRA 3. Necesidades en salud y satisfacción de la demanda. Encuesta CASEN 2000. 
La demanda satisfecha según la afiliación previsional de las personas, mostró niveles similares de satisfacción en el sistema público y privado $(91,7$ y $92 \%$, respectivamente), sin diferencias significativas por sexo.

Por quintiles de ingreso, se observó un incremento de la satisfacción de la demanda hacia los quintiles de mayores ingresos, con diferencias por sexo: las mujeres lograron proporciones de demanda satisfecha levemente menores que los hombres en los quintiles pobres y mayores en los quintiles más ricos, aunque sin significancia estadística (Figura 5).

Analizando la satisfacción de la demanda según el nivel de ingresos en cada sistema previsional, ésta supera el $90 \%$ para todos los grupos por quintiles y por sistemas de salud, sin diferencias. Es decir, no hay relación entre los ingresos de la persona y el sistema de salud al cual pertenece para obtener satisfacción de su demanda de salud en el análisis crudo.

Otra relación analizada, fue la satisfacción de la demanda en función del sistema de servicios utilizados cruzados para los sistemas FONASA e ISAPRE (Figura 6). Entre 12 y 17\% de los adscritos a ISAPRE se atendieron en el sistema público, con los valores más bajos en los quintiles extremos y el máximo en el tercer quintil. Para los adscritos a FONASA que se atendieron en el sistema privado, la curva muestra un marcado ascenso de la proporción desde $6 \%$ en el quintil I a $52 \%$ en el quintil V.

Demanda no satisfecha de atención en salud. La demanda no satisfecha corresponde a $8,2 \%$ de la demanda expresada (Figura 3) y las causas anali-
FIguRA 4. Demanda expresada por edad y sexo. Encuesta CASEN 2000.

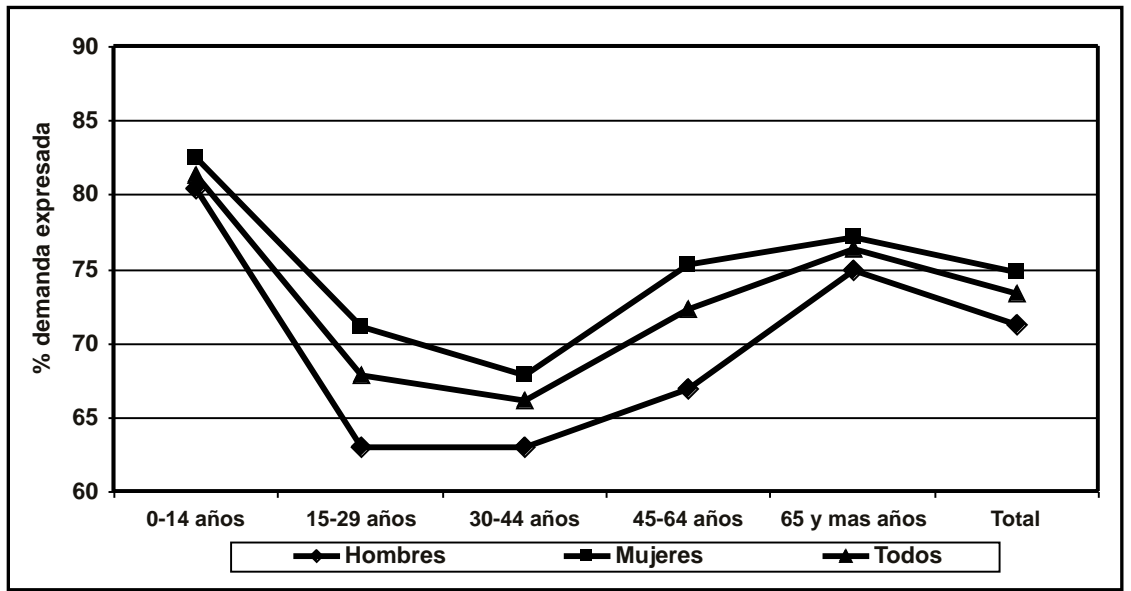

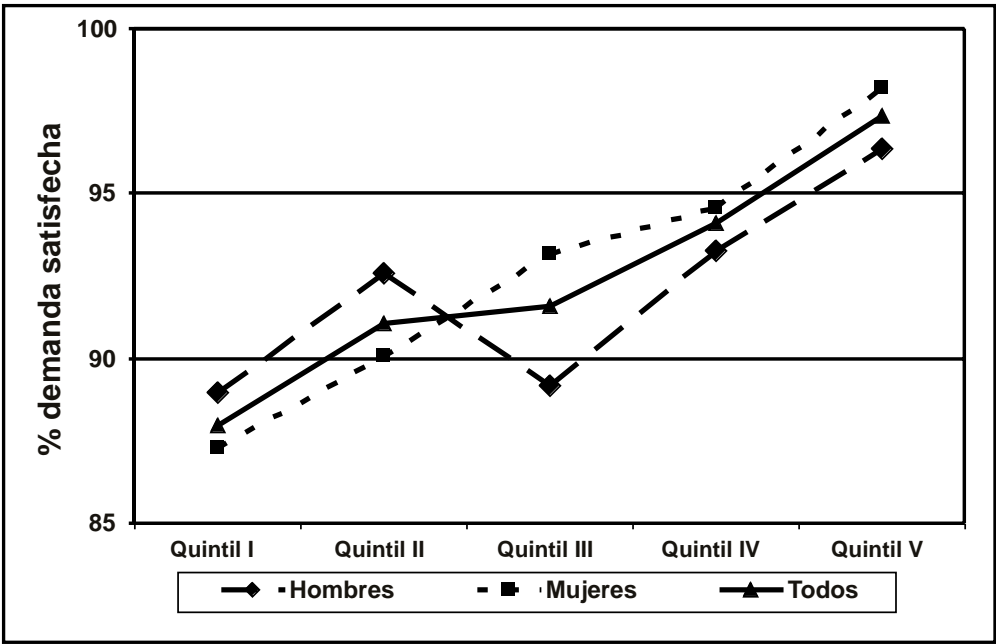

FIgURA 5. Demanda satisfecha por quintil de ingreso y sexo. Encuesta CASEN 2000. 
zadas para no obtener servicios de salud fueron: 1. Barrera financiera: $5,5 \%$. 2. Barrera geográfica: $1,1 \%$. 3. Rechazo del sistema de salud: $1,4 \%$.

Al interior del grupo de demanda no satisfecha, las razones para no tener acceso al sistema de salud correspondieron a $68,8 \%$ debido a barreras financieras, $17,5 \%$ por rechazo del sistema, y $13,7 \%$ por barreras geográficas. Las características de la edad y sexo de la población que no logró consultar en el sistema y su distribución por quintil de ingresos de salud se observan en la Tabla 3, mostrando mayor proporción en las personas de 35 a 64 años, especialmente mujeres. Entre los varones, son los menores de 14 años los que no llegan a la consulta médica. Según sistema previsional en salud, 65,4\% de esta población pertenece a FONASA, 15\% a ISAPRE y $19,4 \%$ no tiene sistema previsional.

El análisis en este mismo grupo según su previsión de salud, muestra que la barrera financiera fue de mayor relevancia en las ISAPRE, 95\% de los casos y en las personas con otras formas de previsión en salud, 82,1\%. En cambio, el rechazo del sistema y las barreras geográficas son proponcionalmente más relevantes en el sistema público de salud. Por quintil de ingresos (Tabla 4), las observaciones son similares, en los quintiles más ricos, el problema es de barrera financiera o de rechazo del sistema principalmente. En el quintil I, pesa la barrera financiera, pero el acceso geográfico y el rechazo del sistema son importantes también.

Análisis multivariado. Siguiendo el esquema de análisis presentado en la Figura 3, se aplicó un modelo de regresión logística, cuya variable de- pendiente es "satisfacción de la demanda". Las variables independientes que se incorporaron en este análisis fueron: edad, sexo, sistema previsional, quintil de ingresos, zona de residencia urbana 0 rural, pertenencia a etnias. La Tabla 5 muestra cada una de las variables y los parámetros de comparación utilizados.

En el modelo de regresión stepwise aplicado las variables ingresan en el siguiente orden: sistema previsional; tramos de edad; quintil de ingresos; pertenencia a etnias (declara pertenecer a etnia y habla 0 entiende el idioma); zona de residencia (urbana o rural) y sexo. De acuerdo a la definición de variables de contraste especificado en el modelo, la base de comparación fue: hombre, menor de 14 años, quintil V, residente en zona urbana y no perteneciente a etnias.

Controlando por cada una de las variables incorporadas al modelo (Tabla 6), la variable más importante en términos de satisfacción de la demanda es la "edad" (Beta entre 0,787 y 0,967). Dentro de los grupos etáreos comparados, los adultos de 30 a 44 años tuvieron mayor riesgo de no recibir atención de salud (2,6 veces), seguidos por los adultos de 65 años y más, cuyo riesgo de no satisfacer la demanda fue 2,4 veces mayor con respecto a un menor de 0 a 14 años. La segunda variable en importancia corresponde a la "pertenencia a etnia". Las personas que se declararon pertenecientes a etnias tuvieron un riesgo de 2,1 veces de que su demanda de salud no fuera satisfecha. La tercera variable identificada fue el "sistema previsional de salud". Las personas no cotizantes en el sistema de salud tuvieron un riesgo

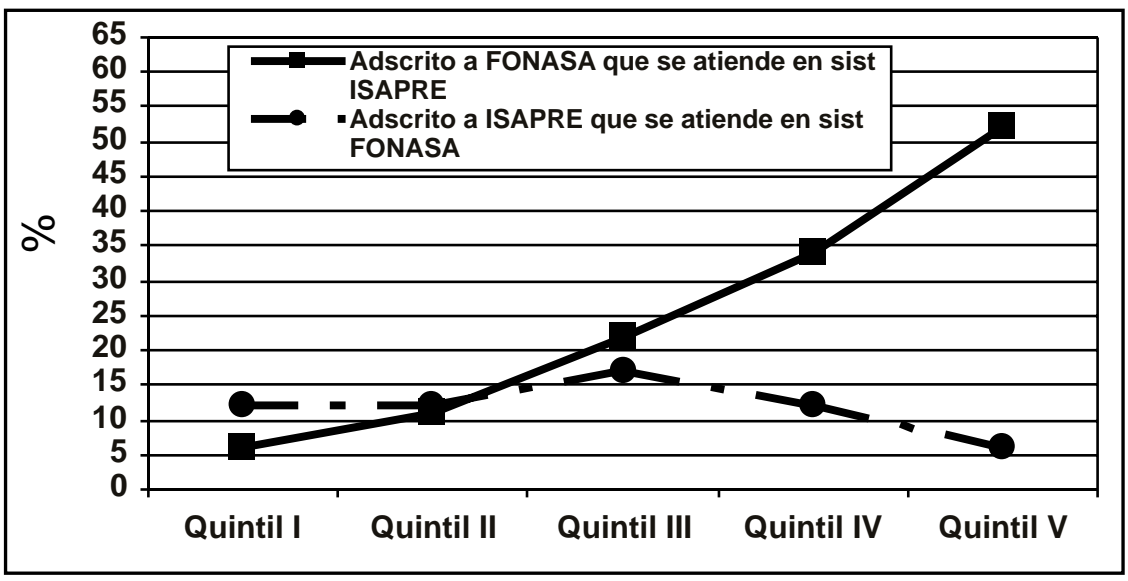

FIgURA 6. Atención de salud según sistema previsional y quintil de ingresos. Encuesta CASEN 2000. 
1,7 veces mayor de no recibir atención en comparación con aquellos afiliados a FONASA o ISAPRE. Los residentes en "zonas rurales" tuvieron un riesgo 1,4 veces mayor de no recibir atención cuando la solicitan, los hombres tuvieron un riesgo levemente mayor 1,1 veces de no ser atendidos cuando tienen un evento de salud o accidente comparados con las mujeres de las mismas características. El "nivel socioeconómico" representado por los quintiles de ingresos fue una variable altamente significativa con una gradiente inversa clara entre ingreso y atención de salud. Las personas del primer quintil (más pobres) tuvieron 2 veces más riesgo de no satisfacer la demanda de salud comparadas con las personas del quinto quintil, diferencia que se reduce a 1,2 al subir hasta el cuarto quintil. Sorprendentemente, cuando se ajusta por las otras variables, las personas pertenecientes a ISAPRE tuvieron 1,5 veces más riesgo de no recibir atención cuando la requieren, comparados con aquellos adscritos a FONASA.

El modelo global de satisfacción de la demanda es altamente significativo explicando más de $70 \%$ de la variabilidad. Las características de la población con mayor riesgo de no satisfacer sus demandas de salud correspondieron, por lo tanto, a los hombres de entre 30 y 44 años, de los hogares más pobres, de zonas rurales, que no tenían sistema previsional y que pertenecían a una etnia.

\section{Discusión}

El presente estudio confirma observaciones señaladas en publicaciones anteriores ${ }^{4,5}$, sobre la distribución diferencial de la población en el sistema de salud público y privado, con selección de la población de mayor riesgo (menores ingresos, mayor edad, mujeres) en el sistema público. Esta distribución fluctúa de acuerdo a las condiciones macroeconómicas del país. En efecto, se observan fluctuaciones de la participación del sistema público y privado en el total de afiliados durante el período comprendido entre 1990 y 2000, con un incremento sostenido de la participación de las ISAPRES en el aseguramiento de la población en la primera mitad, que se revierte en la segunda mitad. El reposicionamiento del sistema público en los últimos años, muy probablemente, se vincula a los efectos que tuvo en Chile la crisis económica intemacional a partir del año 1997, lo que hizo que migrara población de las ISAPRES a FONASA, al dejar de estar cubiertas por falta de empleo o por menores ingresos, que no les permitían comprar los planes de salud privados con su cotización.

Las nuevas preguntas del módulo de salud de CASEN a partir del año 2000, permiten establecer una línea de base con respecto a autopercepción de salud en la población chilena, indicador que no existía y que se correlaciona estrechamente con

Tabla 3. Porcentaje de demanda no satisfecha por sexo y edad. Encuesta CASE N 2000

\begin{tabular}{|lcccccc|}
\hline & $0-14$ años & 15-29 años & 30-44 años & $45-64$ años & 65 y más años & Total \\
\hline Hombres & 22,7 & 26,0 & 18,9 & 21,2 & 11,3 & 100 \\
Mujeres & 9,6 & 16,5 & 29,4 & 28,9 & 15,6 & 100 \\
Todos & 15,1 & 20,5 & 25,0 & 25,6 & 13,8 & 100 \\
& Quintil I & Quintil III & Quintil III & Quintil IV & Quintil V & Total \\
Hombres & 32,0 & 19,9 & 27,8 & 13,9 & 6,4 & 100 \\
Mujeres & 36,9 & 27,6 & 19,3 & 12,8 & 3,4 & 100 \\
Todos & 34,8 & 24,4 & 22,8 & 13,2 & 4,7 & 100 \\
\hline
\end{tabular}

Tabla 4. D emanda no satiffecha: distribución por quintil de ingresos y causas. Encuesta CASEN 2000

\begin{tabular}{|lcccccc|}
\hline & Quintil I \% & Quintil II \% & Quintil III \% & Quintil IV \% & Quintil V \% & Todos \% \\
\hline Falta acceso financiero & 58,8 & 69,7 & 79,3 & 73,9 & 72,6 & 68,8 \\
Falta de acceso geográfico & 19,0 & 13,1 & 8,9 & 11,9 & 6,1 & 13,7 \\
Rechazo del sistema & 22,2 & 17,2 & 11,8 & 14,2 & 21,3 & 17,5 \\
Total & 100,0 & 100,0 & 100,0 & 100,0 & 100,0 & 100,0 \\
\hline
\end{tabular}


Tabla 5. Variables incluidas en el modelo de regresión logística para demanda satisfecha. Encuesta CASEN 2000.

\begin{tabular}{|c|c|c|c|c|c|c|}
\hline \multirow[b]{2}{*}{ Variables } & \multirow[b]{2}{*}{ Categorías } & \multirow[b]{2}{*}{ Frecuencia } & \multicolumn{4}{|c|}{$\begin{array}{l}\text { Códigos parámetros } \\
\text { de comparación }\end{array}$} \\
\hline & & & 1 & 2 & 3 & 4 \\
\hline \multirow[t]{5}{*}{ Edad } & 0-14 años & 6.505 & 0 & 0 & 0 & 0 \\
\hline & 15-29 años & 3.781 & 1 & 0 & 0 & 0 \\
\hline & 30-44 años & 4.501 & 0 & 1 & 0 & 0 \\
\hline & 45-64 años & 5.936 & 0 & 0 & 1 & 0 \\
\hline & 65 y más años & 4.283 & 0 & 0 & 0 & 1 \\
\hline \multirow[t]{2}{*}{ Sexo } & Hombre & 10.376 & 1 & & & \\
\hline & Mujer & 14.630 & 0 & & & \\
\hline \multirow[t]{3}{*}{ Sistema previsional de salud } & Público & 20.531 & 1 & 0 & & \\
\hline & Privado & 3.003 & 0 & 0 & & \\
\hline & Ninguno & 1.472 & 0 & 1 & & \\
\hline \multirow[t]{5}{*}{ Quintil de ingreso autónomo } & I & 8.295 & 1 & 0 & 0 & 0 \\
\hline & II & 6.282 & 0 & 1 & 0 & 0 \\
\hline & III & 4.673 & 0 & 0 & 1 & 0 \\
\hline & IV & 3.605 & 0 & 0 & 0 & 1 \\
\hline & V & 2.151 & 0 & 0 & 0 & 0 \\
\hline \multirow[t]{2}{*}{ Zona de residencia } & Urbano & 15.240 & 0 & & & \\
\hline & Rural & 9.766 & 1 & & & \\
\hline \multirow[t]{2}{*}{ Etnia } & Pertenece & 1.113 & 0 & & & \\
\hline & No pertenece & 23.942 & 1 & & & \\
\hline
\end{tabular}

Tabla 6. M odelo de regresión logística para la demanda de salud. Encuesta CASEN 2000

\begin{tabular}{|c|c|c|c|c|c|c|c|c|}
\hline & B & S.E. & $\begin{array}{l}\text { Wald } \\
\text { B }\end{array}$ & $\begin{array}{l}\text { Df S. } \\
\text { E. }\end{array}$ & $\begin{array}{l}\text { Sig. } \\
\text { Wald }\end{array}$ & $\begin{array}{c}\operatorname{Exp}(\mathrm{B}) \\
\text { Odds ratio }\end{array}$ & \multicolumn{2}{|c|}{ I.C. $\operatorname{Exp}(\mathrm{B}) 95 \%$} \\
\hline Quintil de ingreso (1) & 0,684 & 0,009 & 6389,5 & 1 & 0,000 & 1,98 & 1,95 & 2,02 \\
\hline Quintil de ingreso (2) & 0,555 & 0,008 & 4380,8 & 1 & 0,000 & 1,74 & 1,71 & 1,77 \\
\hline Quintil de ingreso (3) & 0,529 & 0,008 & 4343,0 & 1 & 0,000 & 1,70 & 1,67 & 1,72 \\
\hline Quintil de ingreso (4) & 0,355 & 0,008 & 1890,4 & 1 & 0,000 & 1,43 & 1,40 & 1,45 \\
\hline Sexo (1) & 0,134 & 0,005 & 850,2 & 1 & 0,000 & 1,14 & 1,13 & 1,15 \\
\hline Sistema previsional público/FONASA & & & 32825,1 & 2 & 0,000 & & & \\
\hline Sistema previsional privado/ISAPRE (1) & $-0,728$ & 0,006 & 14252,9 & 1 & 0,000 & 0,48 & 0,48 & 0,49 \\
\hline Sistema previsional otro (2) & 0,512 & 0,009 & 3604,7 & 1 & 0,000 & 1,67 & 1,64 & 1,70 \\
\hline Zona de residencia (1) & 0,322 & 0,007 & 2438,2 & 1 & 0,000 & 1,38 & 1,36 & 1,40 \\
\hline Edad 0-14 años & & & 22785,9 & 4 & 0,000 & & & \\
\hline Edad 15-29 años (1) & 0,856 & 0,007 & 13502,3 & 1 & 0,000 & 2,35 & 2,32 & 2,39 \\
\hline Edad 30-44 años (2) & 0,967 & 0,007 & 18819,5 & 1 & 0,000 & 2,63 & 2,59 & 2,67 \\
\hline Edad 45-64 años (3) & 0,787 & 0,007 & 12239,4 & 1 & 0,000 & 2,20 & 2,17 & 2,23 \\
\hline Edad 65 y mas años (4) & 0,886 & 0,008 & 12363,3 & 1 & 0,000 & 2,42 & 2,39 & 2,46 \\
\hline Etnia & 0,757 & 0,012 & 3999,8 & 1 & 0,000 & 2,13 & 2,08 & 2,18 \\
\hline Constante & $-2,458$ & 0,008 & 85345,8 & 1 & 0,000 & 0,09 & & \\
\hline
\end{tabular}


mortalidad y morbilidad ${ }^{6-13}$. Al respecto, se observa que los jóvenes tienen mejor autopercepción de salud que las personas de mayor edad y los hombres mejor que las mujeres en todas las edades. Los quintiles de mayores ingresos muestran mejor autopercepción de salud, lo que también se observa al analizar las regiones donde la curva de mala percepción de salud sigue la misma distribución de la proporción de población en situación de pobreza, situación ya descrita en un estudio previo efectuado por nosotros14. Estos resultados son similares a otros reportados en estudios de autopercepción en países desarrollados ${ }^{15-19}$.

En cuanto a frecuencia de eventos de salud en los 30 días previos, un porcentaje relativamente pequeño de la población $(12,9 \%)$ sufrió un evento y de manera coherente con la autopercepción, la frecuencia de problemas de salud es más alta en los quintiles de menos ingresos, en las mujeres y en los grupos etáreos de 0 a 14 años y de 45 a 64 años en ambos sexos. Es especialmente elevada la percepción de problemas de salud en los varones de 0 a 14, lo que probablemente se asocie a una mayor percepción, de las madres, de enfermedad en los hijos varones, especialmente en menores de dos años, hecho que habíamos demostrado en estudios anteriores ${ }^{20}$.

En cuanto a la demanda expresada, aunque los grupos etáreos de mayor necesidad fueron los menores de 14 años y los mayores de 65 en ambos sexos, la demanda fue mayor en las mujeres en todas las edades. También fue más frecuente la expresión de la demanda entre los quintiles de mayores ingresos, situación que puede reflejar un mayor nivel de preocupación por el estado de salud frente a la percepción de un evento.

La demanda satisfecha alcanzó a casi $92 \%$ de la demanda expresada en el análisis bivariado, con probabilidades aparentemente similares en los dos sistemas de previsión de salud para todos los grupos según ingresos, sin embargo, en el modelo de regresión ajustado, se observa que la adscripción a FONASA representa un factor "protector" y tienen mayor probabilidad, casi 2 veces, de recibir servicios de salud, cuando se ajusta por las otras variables.

En cuanto a causas de falta de acceso a atención de salud, la causa más frecuente fue la barrera financiera, para todos los quintiles y tipos de previsión de salud. La falta de accesibilidad geográfica y el rechazo del sistema de salud fueron causas crecientes en importancia a medida que disminuía el quintil de ingreso, lo que constituye una diferencia injusta y evitable, es decir, una clara inequidad en el acceso a atención de salud en el país.

El análisis multivariado confirmó lo encontrado, los factores asociados a falta de acceso fueron: edad sobre 14 años, pertenecer a una etnia, adscripción a ISAPRE, residencia en área rural, pertenecer al quintil de menores ingresos y sexo masculino, variables que explican más de $70 \%$ de la variabilidad del modelo. Aunque de pequeña magnitud, el mayor riesgo de los hombres de no recibir atención de salud es una situación que merece mayor atención para identificar las causas, que pueden relacionarse con barreras de acceso tales como los horarios de atención en los centros de salud u otras barreras como factores culturales que deberían modificarse en relación con su propia salud.

El "pertenecer a una etnia" se analizó para los que declararon pertenecer a etnias originarias y además hablar o entender una de las lenguas de los pueblos originarios existentes en Chile, grupo que muestra un riesgo 2,1 veces mayor de que la demanda de salud no sea satisfecha. Esta característica se explora poco en los análisis de nuestra población, pero con frecuencia aparece como un factor de riesgo, el que se asocia además a pobreza, lo que constituye un desafío para refocalizar las acciones del sector salud para incluir de manera preferencial a estos grupos.

El presente estudio destruye uno de los prejuicios más comunes en la opinión pública, con relación a que el sector privado responde mejor a los requerimientos de atención que el público. Por el contrario, nuestros datos demuestran que cuando se ajusta por las otras variables que inciden en la satisfacción de la necesidad de salud, hay mayor probabilidad de tener el servicio para los afiliados al sistema público.

Las inequidades encontradas deberían ser corregidas por las actuales leyes propuestas en la reforma, especialmente aquellas relacionadas con el plan AUGE y con la reformulación de los mecanismos de financiamiento de las ISAPRES desde un seguro individual hacia un sistema de seguridad social.

Finalmente, el estudio muestra el avance que ha significado el rediseño del módulo de salud de CASEN, en términos de permitir realmente el monitoreo del acceso a atención médica por parte 
de la población chilena. Es importante que el nuevo módulo se mantenga en las versiones futuras de CASEN, para permitir, a través del análisis de tendencias, el monitorear efectivamen-

\section{REFERENCIAS}

1. Miranda E. La Salud en Chile. Evolución y Perspectivas. Santiago: Centro de Estudios Públicos; 1994.

2. Documentos Reforma de Salud: Ministerio de Salud "Hacia un nuevo modelo de gestión en salud", Contenidos del Proyecto de Ley de Autoridad Sanitaria y Gestión en Salud y de Medidas Administrativas Inmediatas. Junio 2002. Ley de derechos y deberes, Plan AUGE, Ley de ISAPRES.

3. Encuesta CASEN, instrumento y metodología en la página web de MDEPLAN: http://www.mideplan. $\mathrm{cl} /$ sitio/Sitio/casen/htm/casen.htm.

4. Impacto de las Políticas Públicas en la Situación de Salud, 1990-1996. Gobierno de Chile, Ministerio de Planificación y Cooperación, División Social, Departamento de Estudios Sociales. Diciembre, 1998. http://www.mideplan.cl/estudios/ salud01.pdf.

5. Análisis de la VIII Encuesta de Caracterización Socioeconómica Nacional (Casen 2000) Documento № 5. Situación de la Salud en Chile, 2000. Gobierno de Chile, Ministerio de Planificación y Cooperación. http://www.mideplan.cl/sitio/Sitio/ estudios/documentos/salud2000.pdf.

6. BaKer M, Stabile M, Deri C. What Do Self-Reported, Objective, Measures of Health Measure? University of Toronto, June 2002.

7. Mackenbach JP, Kunst AE, CavelaArs AE, GroenhoF F, GeURTS JJ. Socioeconomic inequalities in morbidity and mortality in western Europe. The EU Working Group on Socioeconomic Inequalities in Health. Lancet 1997; 349: 1655-9.

8. KunTS A, MackenBaCH JP. Measuring socioeconomic inequalities in health. WHO Regional Office for Europe, EUR/ICP/RPD 416, 1995.

9. Self-Reported Health Status: Social and Demographic Characteristics. Washington State Population Survey. Research Brief № 2, June 1999.

10. Dalstra JA, Kunst AE, Geurts JJ, Frenken FJ, MaCKENBACH JP. Trends in socioeconomic health te si la reforma de salud cumplirá uno de sus objetivos más ambiciosos, cual es el transformarse en un instrumento de equidad de acceso a atención de salud en Chile.

inequalities in the Netherlands, 1981-1999. J Epidemiol Community Health 2002; 56: 927-34.

11. Mackenbach JP, Cavelaars AE, Kunst AE, Groenhof F. Socioeconomic inequalities in cardiovascular disease mortality; an international study. Eur Heart J 2000; 21: 1141-51.

12. KunST AE, MackenBaCh JP. The size of mortality differences associated with educational level in nine industrialized countries. Am J Public Health 1994; 84: 932-7.

13. Kunst AE, GeuRTs JJ, van den Berg J. International variation in socioeconomic inequalities in self reported health. J Epidemiol Community Health 1995; 49: 117-23.

14. Subramanian V, Delgado I, Jadue L, Kawachi I, Vega $\mathrm{J}$. Inequidad de ingreso y autopercepción de salud: un análisis desde la perspectiva contextual en las comunas chilenas. Rev Méd Chile 2003; 131: 321-30.

15. Van Doorsiaer E, Jones A. Inequalities in selfreported health: validation of a new approach to measurement. Economic determinants of the distribution of health and health care in Europe. Ecuity II Project. European Community Biomed II program. January 2002.

16. KenNedy BP, Kawachi I, Glass R, Prothrow-Stith D. Income distribution, socioeconomic status, and self rated health in the United States: multilevel analysis. BMJ 1998; 317: 917-21.

17. Lahelma E, Manderbacka K, RahKonen O, Karisto A. Comparisons of inequalities in health: evidence from national surveys in Finland, Norway and Sweden. Soc Sci Med 1994; 38: 517-24.

18. WAGSTAFF A ET AL. On the measurement of inequalities in health. Soc Sci Med 1991; 33: 545-57.

19. Stronks K, van de Mheen H, van den Bos J, MACKENBACH JP. Smaller socioeconomic inequalities in health among women: the role of employment status. Int J Epidemiol 1995; 24: 559-68.

20. Vega J, Bedregal P, Jadue L, Delgado I. Equidad de Género en el Acceso a la Atención de Salud en Chile. Rev Méd Chile 2003; 131: 669-78. 